\title{
Full Modal Analysis of the Brillouin Gain Spectrum of an Optical Fiber
}

\author{
Luca Tartara \\ Department of Electronics, Università degli Studi di Pavia, Via Ferrata 1, 27100 Pavia, Italy \\ Tel: (39) 0382 985595, Fax: (39) 0382 422583, e-mail: luca.tartara@unipv.it \\ Christophe Codemard, Jean-Noel Maran* \\ Optoelectronics Research Centre, University of Southampton, Highfield, Southampton SO17 1BJ, United \\ Kingdom \\ *now with Coractive, 2700 Jean-Perrin, Quebec (Qc), Canada \\ Rim Cherif, Mourad Zghal \\ Cirta'Com Laboratory, Engineering School of Communication of Tunis (Sup'Com), Ghazala Technopark, \\ 2083 Ariana, Tunisia
}

\begin{abstract}
We present a numerical study of stimulated Brillouin scattering in optical fibers based on a full modal analysis of the acoustic and optical properties. The computation of each acoustic mode supported by the fiber structure allows us a deep and detailed investigation of the characteristics of the Brillouin gain spectrum. We focus our attention on optical fibers acting as acoustic antiwaveguides where the biggest contribution to the Brillouin response often comes from very high-order modes but it is sometimes overlooked because of computational issues. Our analysis clearly highlights their role and their dependence on the physical and geometrical structure of the fiber.
\end{abstract}

\section{INTRODUCTION}

Fiber lasers are nowadays emerging as the most powerful solid-state laser technology because of their compactness, their reliable and efficient operation, and the high power levels attainable. However several issues degrading the laser performance arise and need to be tackled when increasing the light intensity in the fiber. Stimulated Brillouin scattering (SBS) is often the most detrimental effect for narrow-line lasers [1]. Moreover SBS sets a limit to the power which can be transmitted in a fiber communication system. On the other hand SBS can be conveniently employed for sensing applications and for all-optical signal elaboration. A great deal of research has thus been devoted to the design of fiber geometries in terms of their Brillouin response. The Brillouin gain spectrum (BGS) summarizes all the main properties characterizing a fiber from this point of view and hence is usually the object of the numerical investigations of SBS.

Several approaches have been exploited for the computation of the BGS starting from the modal properties of the fiber $[2,3]$. The usual assumption is that the fiber can support a single optical mode so that only a limited set of axially-symmetric acoustic modes are evaluated. In this paper we present a numerical study of SBS in optical fibers based on a full modal analysis of the acoustic behaviour. The computation of the characteristics of each acoustic mode supported by the fiber structure allows a deep and detailed investigation of the BGS. This is particularly important in the case of optical fibers acting as acoustic antiwaveguides as higher-order modes often provide the biggest contribution to the Brillouin response.

\section{THEORETICAL BACKGROUND}

The characterization of the optical and acoustic properties of the fiber is performed in terms of modal propagation. The acoustic modes of the fiber are obtained by solving the 2-D scalar-wave equation satisfied by the longitudinal displacement field $u(x, y)$ :

$$
\nabla_{t}^{2} u+\left(\frac{\omega_{a c o}^{2}}{v_{l}^{2}}-\beta_{a c o}^{2}\right) u=0
$$

where $\nabla_{t}^{2}$ is the transverse Laplacian operator in the $(x, y)$ plane, $\omega_{a c o}$ is the angular frequency of the acoustic wave, $v_{l}$ is the longitudinal acoustic velocity, and $\beta_{a c o}$ is the propagation constant of the mode. The longitudinal acoustic velocity varies on the cross-section of the fiber according to the type and concentration of dopants.

The solution to Eq. (1) yielding the modal field patterns along with the corresponding propagation constants is obtained by employing a finite-element method (FEM) solver working on a mesh made up of $\approx 10^{4}$ elements. 
The equation is firstly solved for different values of the frequency and evaluating the corresponding phase velocity for each mode. In such a way it is possible to obtain the dispersion relations to be used to determine the resonance frequency. In fact the back-scattering process is effective only when the phase-matching condition $\beta_{\text {opt }}$ $=2 \beta_{a c o}$ is fulfilled with $\beta_{\text {opt }}$ being the propagation constant of the optical mode. The Brillouin frequency shift $f_{B}$ is then:

$$
f_{B}=\frac{2 n_{e f f} v_{e f f}}{\lambda}
$$

where $\lambda$ is the optical wavelength in vacuum, $n_{\text {eff }}=\beta_{\text {opt }} \lambda /(2 \pi)$ is the effective index of the optical mode, and $v_{\text {eff }}$ $=\omega_{a c o} / \beta_{a c o}$ is the effective velocity of the longitudinal acoustic mode interacting with the optical mode.

The effective index is obtained from the 2-D scalar wave equation for the electric field $E(x, y)$ :

$$
\nabla_{t}^{2} E+\left(\frac{2 \pi}{\lambda}\right)^{2}\left(n^{2}-n_{e f f}^{2}\right) E=0
$$

where $n$ is the refractive index being dependent on the transverse coordinates because of the contribution of the different dopants.

Eq. (3) can be solved by means of the same algorithm implemented for Eq. (1) yielding not only the effective index to be used in Eq. (2), but also the spatial pattern of the optical field $E(x, y)$.

Once the effective index for the optical mode has been evaluated, eq. (2) is used to determine the frequency at which the Brillouin scattering occurs for each acoustic mode. Eq. (1) is then solved for such value of the frequency in order to obtain the spatial profile of the displacement field. The modal distributions of both the optical and the acoustic field determine then the efficiency of the back-scattering process according to the value of the overlap integral:

$$
I=\frac{\left(\int|E|^{2} u^{*} d x d y\right)^{2}}{\int|E|^{4} d x d y \cdot \int|u|^{2} d x d y}
$$

The integral is numerically evaluated on the cross-section of the fiber and it is normalized so that its value is not greater than unity. It is worth to note that the maximum overlap does not occur when the two fields have the same spatial pattern as the integral in the numerator of eq. (4) implies.

The next step leading to the evaluation of the BGS is the computation of the contribution of each acoustic mode given by:

$$
s(f)=\frac{4 \pi n_{e f f}^{8} p_{12}^{2}}{\lambda^{3} \rho c f_{B} \Delta f_{B}} I \frac{\left(\frac{\Delta f_{B}}{2}\right)^{2}}{\left(f-f_{B}\right)^{2}+\left(\frac{\Delta f_{B}}{2}\right)^{2}}
$$

where $p_{12}$ is the photoelastic constant, $\rho$ is the material density, $\mathrm{c}$ is the speed of light, and $\Delta f_{B}$ is the full-width at half maximum bandwidth of the resonance, which is assumed to be $40 \mathrm{MHz}$ for every acoustic mode. As the modes are statistically independent, the BGS is finally given by the sum of each contribution as given by eq. (5).

\section{RESULTS}

Our simulation model can be employed for the evaluation of the BGS of any kind of fiber. Because of the role of SBS as the main limiting factor for high-power fiber lasers, we have focused our investigation on double-clad fibers and particularly on fiber structures acting like antiwaveguides for the acoustic field. Such fibers have indeed drawn much interest because of their capacity of increasing the SBS threshold. In fact the lower-order acoustic modes are confined in the outer core or in the cladding while the optical mode propagates in the inner core. The overlap integral defined by eq. (4) has then a low value decreasing the Brillouin gain coefficient as proved by eq. (5). However a great number of acoustic modes interact with the optical field with a set of higherorder modes being confined to some extent in the inner core region. Therefore these modes often provide the most substantial contribution to the BGS because of their spatial overlap to the optical field. Nevertheless such a contribution is sometimes overlooked in numerical investigations as several computational issues arise in the 
analysis of those modes because of the very high order. Our approach based on a full modal characterization of the acoustic behaviour of the fiber allows us to highlight every aspect of the back-scattering process.

We report here the results of the BGS computation for a recently proposed type of fiber characterized by the use of the two different dopants in the optical core region having a different effect on the optical and acoustic properties of pure silica [4]. In such a way it is possible to achieve a step-index profile in the whole core region, while the acoustic velocity is higher in the inner part of the core and lower in the outer part assuming a so-called W-shape. This geometry can be realized by employing $\mathrm{Al}_{2} \mathrm{O}_{3}$ and $\mathrm{GeO}_{2}$ as dopants: they both increase the refractive index with respect to pure silica, but the former increases also the acoustic velocity, while the latter decreases it. A proper combination of such dopants in the core can thus make the fiber a waveguiding structure as far as lightwave propagation is concerned. However it behaves like an antiwaveguide for the acoustic field with an intermediate velocity in the cladding.

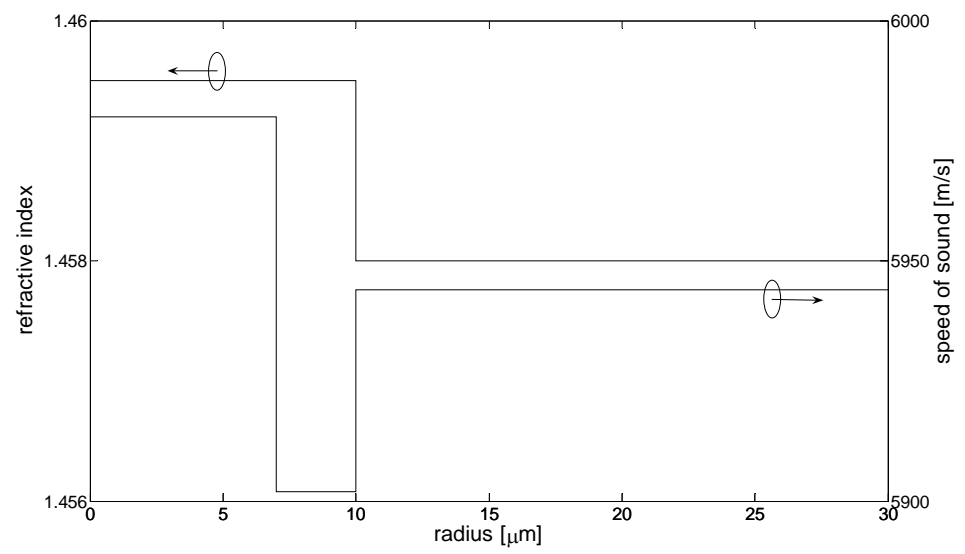

Figure 1. Refractive index and speed of sound profiles used for simulations.

The values of the optical and acoustic parameters used in our simulation are reported in fig. 1 which shows the variation of the refractive index and of the acoustic velocity along the radial coordinate. The optical core radius is $10 \mu \mathrm{m}$ so that the fiber is slightly multimodal at the wavelength of $1064 \mathrm{~nm}$ used in the computation of the BGS. A great deal of attention is devoted to the influence of the cladding size. In fact, as the cladding diameter is increased in order to accommodate higher pump power levels, a higher number of acoustic modes can propagate. Consequently higher-order modes acquire a greater influence on the Brillouin response of the fiber and clearly emerge as the main limitation.

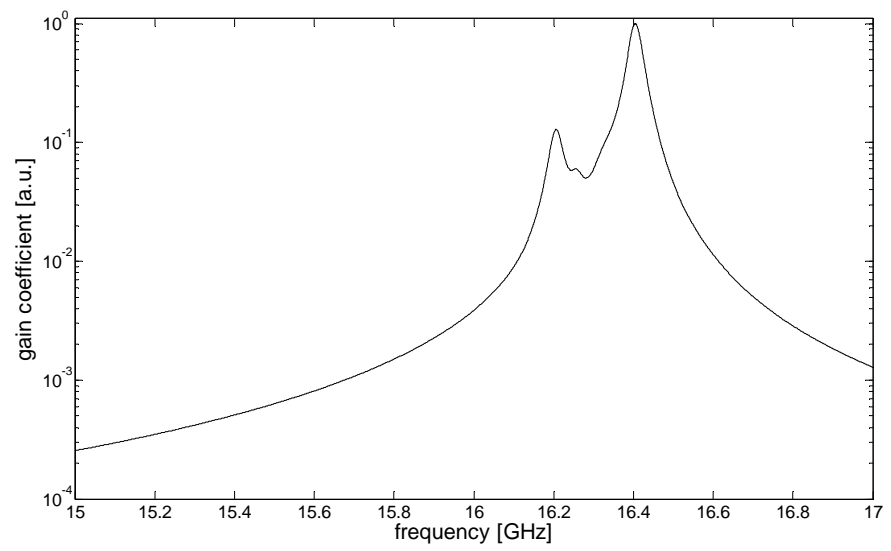

Figure 2. Brillouin gain spectrum for a cladding radius of $62.5 \mu \mathrm{m}$.

In fig. 2 we report the outcome of the numerical computation for a cladding radius of $62.5 \mu \mathrm{m}$ when the optical field propagates as the fundamental mode. The BGS is characterized by two peaks around the acoustic frequencies of $16.2 \mathrm{GHz}$ and $16.4 \mathrm{GHz}$ respectively. The first peak has to be ascribed to the action of lowerorder modes, while higher-order modes are responsible for the second peak. In fact, the higher the order of the 
mode, the higher its phase velocity, and from eq. (2) the bigger the Brillouin frequency shift. A further insight into the BGS of fig. 2 is provided by the graph of fig. 3 showing the value of the overlap integral for each mode. A set of several modes having order from 3150 to 3300 give significant contributions to the Brillouin backscattering process with the mode of order 3228 yielding the maximum spatial overlap. On the other hand the value of the overlap integral for the fundamental mode is 4 times lower and only a very few other lower-order modes interact to an appreciable extent with the optical field as the inset in fig. 3 shows. This clearly explains the two peaks of the spectrum: the one at a lower frequency is due to the lower-order modes, the one at a higher frequency is due to the higher-order modes. The greater spatial overlap along with the greater number of interacting modes makes the latter contribution the dominant one.

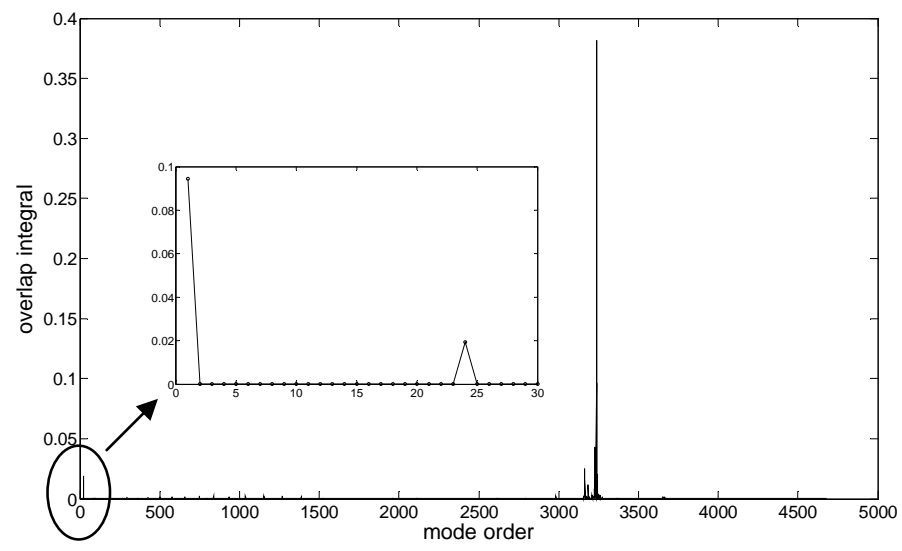

Figure 3. Values of the overlap integrals for a cladding radius of $62.5 \mu \mathrm{m}$.

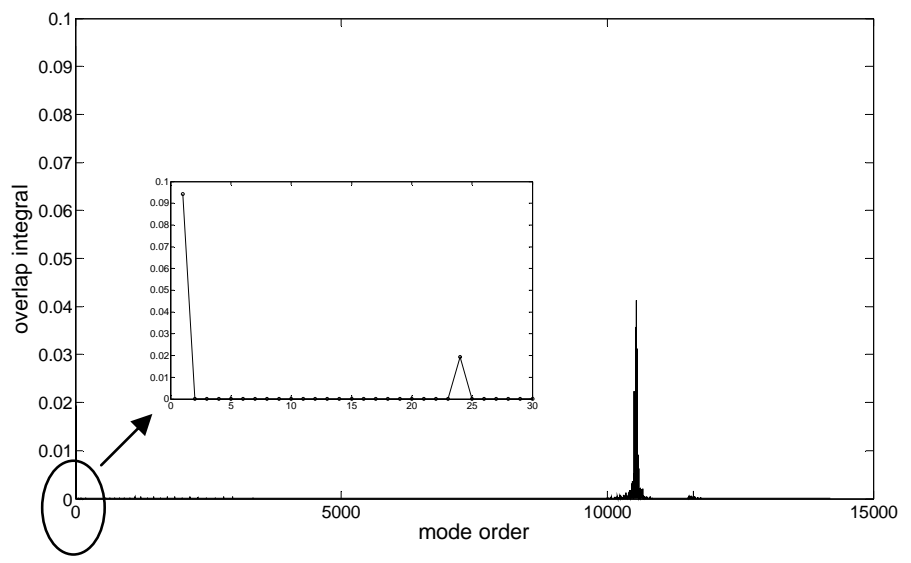

Figure 4. Values of the overlap integrals for a cladding radius of $125 \mu \mathrm{m}$.

We have repeated the computation for a doubled value of the cladding radius. The BGS has almost no difference with respect to the one obtained for a smaller cladding size. However the modal analysis as summarized by the values of the overlap integrals of fig. 4 reveals a different scenario as far as the contribution of higher-order modes is concerned. The maximum value of the overlap integral which is provided by mode 10546 is indeed one order of magnitude smaller than the maximum value obtained for a cladding size of 62.5 $\mu \mathrm{m}$ and on the average the degree of spatial overlap of higher-order modes is significantly reduced. However the number of modes providing a not negligible contribution to the BGS is higher because of a larger size of the structure. As such modes have resonance frequencies very close to each other, the overall effect in the BGS is a peak being in the same position. The computation then provides an intensity of the Brillouin response having the same magnitude so that the higher-frequency part of the spectrum is unchanged. Also the effect of lower-order modes is to give rise to an identical contribution in the BGS, but in this case the modal analysis reveals that the peak at a lower-frequency is originated by the same modes as for the fiber having a smaller cladding diameter. The overlap integrals calculated for these modes have of course the same values as it can be seen by the comparison of the insets of fig. 3 and fig. 4. The same results have been obtained for several values of the 
cladding radius ranging from $20 \mu \mathrm{m}$ to $200 \mu \mathrm{m}$. The conclusion which can be drawn is that the cladding size has almost no influence on the BGS. As far as lower-order modes are concerned, the reason is that these modes are confined in the outer core and so the extent of the cladding has a negligible effect. The properties of higherorder modes are instead dependent on the cladding radius as they spread on the whole fiber cross-section. The effect is twofold: a higher number of modes can be supported by a larger cladding but their extension into the inner core region is comparatively smaller just because of a bigger size of the cladding. The former effect would increase by itself the Brillouin response but the latter has the opposite influence because of the consequent smaller spatial overlap to the optical field. The two effect turn out to compensate each other so that the BGS is practically independent of the cladding size.

\section{CONCLUSIONS}

We have proposed a method for the computation of the Brillouin gain spectrum of an optical fiber relying on the numerical evaluation of each acoustic mode. This approach is particularly useful for the characterization of antiwaveguiding structures where higher-order modes have been shown to play a fundamental role. We have also investigated the influence of the cladding radius and found out that the Brillouin gain spectrum can be treated as independent of the cladding size.

\section{REFERENCES}

[1] G.P. Agrawal, Nonlinear Fiber Optics, New York: Academic Press, 2006.

[2] Y. Koyamada, et al.: Simulating and Designing Brillouin Gain Spectrum in Single-Mode Fibers, $J$. Lightwave Technol., vol. 22. pp. 631-639, Feb. 2004.

[3] W. Zou, et al: : Acoustic modal analysis and control in $w$-shaped triple-layer optical fibers with highlygermanium-doped core and F-doped inner cladding, Opt .Expr., vol. 16. pp. 10006-10017, July 2008.

[4] M.J. Li, et al.: Al/Ge co-doped large mode area fiber with high SBS threshold, Opt .Expr., vol. 15. pp. 8290-8299, July 2007. 